\title{
Cuando la realidad supera a la ficción. Los Naufragios de Alvar Núñez Cabeza de Vaca
}

\author{
ALFREDO JIMÉNEZ NÚÑEZ \\ Dpto. de Antropología Social \\ Universidad de Sevilla
}

\section{RESUMEN}

El autor se refiere a la Relación que escribió Alvar Núñez Cabeza de Vaca a poco de su primer regreso de América - popularmente conocida como Naufragios- como un texto básico para plantear la frágil línea que delimita la literatura y la realidad. A partir de dicha fuente destaca el posible valor de los textos literarios como documentos etnográficos útiles para el análisis antropológico, así como la cuestión de la veracidad y credibilidad de los textos, la diferencia entre realidad y ficción, si sólo puede ser verdad y creíble lo que es real, y si sólo los hechos reales pueden ser objeto de la antropología. Como una manera de llevar hasta sus extremos estos condicionantes, propone la posibilidad de que la realidad supere a la ficción y resulte a primera vista menos creíble que la propia ficción.

Palabras clave: Etnoliteratura, Alvar Núñez Cabeza de Vaca.

\section{SUMMARY}

In order to pinpoint the thin line separating literature from reality, the author makes reference to Alvar Núnez Cabeza de Vaca's Relación, popularly known as Naufragios, which the famous explorer wrote sometime after his first returning from America. Jiménez Núñez suggests the convenience of using literary texts as ethnographic documents in anthropological analysis. In addition, he touches upon related issues, such as the veracity and credibility of the literary texts, the difference between fact and fiction, and whether or not only facts are suitable objects for analysis in anthropology. In an attempt to take his position to its limit, he reminds us that facts can overcome fiction and thereby become, at first sight, even less credible than fiction.

Key words: Ethnoliterature, Alvar Núñez Cabeza de Vaca.

El primer seminario sobre etnoliteratura, celebrado en la Universidad de Córdoba en noviembre de 1993, me ofreció la oportunidad de ir un poco más atrás, o un poco más allá, del tema concreto de un encuentro que tenía por objeto explorar las posibilidades de la obra literaria como

RDTP, LX, 1 (2005): 109-121 
fuente etnográfica. La elaboración de la ponencia que sirvió de marco al seminario me llevó a repasar mis propios textos y a leer o releer otros en relación al lugar de la antropología dentro de las ciencias sociales, sus conexiones con las humanidades, y su propia definición como ciencia a la vista de su objeto de estudio, sus fuentes, métodos y técnicas (Jiménez Núñez 1994). Dediqué especial atención a la etnohistoria, o antropología histórica, como método basado en el uso sistemático de una adecuada, abundante y diversa documentación de archivo. A partir de la posibilidad de la etnohistoria como método antropológico — posibilidad que hoy ya no se cuestiona-, analicé la situación de la etnografía a través de lo que se había escrito sobre el tema en los últimos años. La crisis de la antropología - particularmente del trabajo de campo- me hizo reflexionar sobre otras fuentes de conocimiento y otros métodos que pedían paso, como hace unas décadas lo pedía la etnohistoria. En resumen, las severas críticas caídas sobre grandes figuras de la antropología de la primera mitad del siglo $\mathrm{xx}$, y la consiguiente desmitificación del trabajo de campo, inclinaban a conceder un margen de confianza a la etnoliteratura como otra vía para penetrar en la complejidad de la conducta humana. Y terminaba mi ponencia con el siguiente párrafo:

\footnotetext{
Una última reflexión interrogante a la vista del confuso y difuso panorama actual de disciplinas y métodos a causa de tanta crisis y tantos juegos de posturas e intenciones: ¿Por qué no intentar nuevos caminos ahora que los más transitados están llenos de amenazantes curvas, aunque también de sugerentes encrucijadas? ¿Por qué no favorecer esas corrientes de convergencia que cada día nos empujan con más fuerza a preguntarnos, casi sabiendo la respuesta de antemano, si la antropología es ciencia social, historia o humanidades? Son interrogantes que nos llevan a cuestionarnos, más que nunca, si la etnografía es pura actividad empírica o, en el fondo y además, es retórica y juegos de lenguaje. Después de todo, ¿no es cierto que la literatura es testimonio y la antropologia es un arte? (Jiménez Núñez 1994: 47).
}

Mi aportación al segundo seminario, celebrado en 1995, fue un ensayo que tenía mucho de reflexión personal. Su título: "La novela como etnografía: del comisario Maigret a Los santos inocentes" (Jiménez Núñez 1997). Me serví de las novelas de Georges Simenon que tienen como protagonista al popular comisario y de la novela de Miguel Delibes para comparar y discurrir sobre la condición humana. Afirmaba yo que había aprendido más sobre los humanos leyendo pura literatura -aunque no cualquier literatura-, que a través de las etnografías y los tratados de teoría antropológica. Admito que lo que se aprende en uno y otro caso son cosas hasta cierto punto distintas y de muy diferente nivel, pero complementarias. Acepto que algunas cuestiones difícilmente se pueden 
probar cuando son esencialmente ficción, mientras que otras se pueden demostrar porque son resultado de una experiencia de campo. Aunque esta distinción nos llevaría de nuevo a cuestionarnos si las cosas son así de claras y rotundas; que creo que no lo son.

Decía yo también en 1995 que para entonces creía en las posibilidades de la etnoliteratura más que cuando el Profesor Manuel de la Fuente Lombo me embarcó por primera vez en esta experiencia metodológica, pero seguía manteniendo mis reservas. Me reafirmaba, sobre todo, en que cualquier literatura no será igualmente apta, ni siquiera utilizable, para la aplicación de un método que ha de reunir, entre otros, los requisitos propios y mínimos de una verdadera labor científica encuadrada, en este caso, en el marco de las ciencias sociales y, por tanto, sujeta a sus exigencias. La crisis del trabajo de campo como fuente empírica de la antropología y su actual proceso de desmitificación no justificarían la aparición de otros métodos y fuentes que participaran de los mismos o peores males. Y esta advertencia debía presidir los intentos que se hicieran en este novedoso campo.

Me planteé como tema de discusión para el tercer seminario sobre etnoliteratura cuestiones como la veracidad y credibilidad de los textos, la diferencia entre realidad y ficción, si sólo puede ser verdad y creíble lo que es real, y si sólo los hechos reales pueden ser objeto de la antropología. Como una manera de llevar hasta sus extremos estos condicionantes, propongo la posibilidad de que la realidad supere a la ficción y resulte a primera vista menos creible que la propia ficción. Sobre la veracidad de los textos etnográficos remito a la obra de Clifford Geertz (1983; 1989) y en cuanto a la verdad, recuerdo la perplejidad de Pilato al escuchar de Cristo que había venido al mundo para dar testimonio de la verdad. Pilato preguntó: "¿Y qué es la verdad?. (Juan, 18, 38). Cada vez creo menos en lo absoluto cuando se trata de la condición humana. Desde la antropología, y en relación con la investigación de campo y sus interpretaciones, podríamos también preguntarnos, ¿qué es la verdad?

Una de las mayores contribuciones que ha hecho la antropología a las ciencias sociales es advertir y demostrar la relatividad de todo lo que es humano. El relativismo cultural es una base y un estímulo para cuestionar y desmontar las visiones etnocéntricas que tanto han favorecido en la historia y en el presente los prejuicios raciales y culturales, la discriminación, la intolerancia, la violencia... A este principio de la relatividad pertenece esa corriente que se llamó la nueva etnografía, que bajo el término de "visión emic trata de conocer y mostrarnos "al otro" desde sus propias circunstancias, en sus propias palabras, según su cosmovisión, y no desde la posición del observador o antropólogo. Pues bien, combi- 
nando el principio de la relatividad y la cuestión de la verosimilitud, he escogido para el presente ensayo a un autor y un texto, lo que me ha situado en un lugar y un tiempo.

Se ha dicho y repetido que a veces la realidad imita al arte. Yo parafraseo esta afirmación al decir que a veces la realidad supera a la ficción. Y si esto es así, ¿cómo podemos clasificar y separar los textos sobre la base de lo que es verdad o es mentira, de lo que es realidad, porque realmente ocurrió, de lo que es ficción porque es creación o invención de un autor? ¿Quién puede decir hasta qué límites el ser humano es capaz de hacer cosas increíbles? ¿Quién tiene capacidad para juzgar en términos absolutos fenómenos que corresponden a la compleja naturaleza del hombre y a su conducta? Me serviré para ilustrar mis reflexiones de un personaje real que vivió una aventura real, de la que nos dio cuenta en un texto que es a la vez etnografía y literatura. Lo primero, porque es descripción de lo observado por el autor mediante una relación larga e íntima con otras gentes, otra cultura. Lo segundo, porque el relato tiene una calidad literaria reconocida por sus muchas ediciones en español y otros idiomas. Me estoy refiriendo a la Relación que escribió Alvar Núñez Cabeza de Vaca a poco de su primer regreso de América, popularmente conocida como Naufragios. Es una obra que participa de varios géneros, pues es historia o narración de unos acontecimientos, es autobiografía y tiene la estructura de una novela de aventuras.

Cabeza de Vaca era de noble y adinerada familia. Nació o se crió en Sevilla. Participó en las guerras de Italia, pero cuando salió de Sanlúcar de Barrameda en el mes de junio de 1527 era muy joven, sin experiencia alguna en las Indias. A pesar de ello, iba de segundo jefe con los cargos de tesorero y alguacil mayor de la expedición mandada por Pánfilo de Narváez para la exploración y colonización de la Florida, descubierta por Ponce de León en su exploración de 1512-1513. La expedición partió de Cuba en la primavera de 1528 , y el desembarco fue, muy probablemente, en la bahía de Tampa. Hombres y caballos marcharon a pie mientras los barcos seguían por la costa en busca de un puerto. Los hombres de tierra no volverían a encontrarse con la armada. Las noticias de los indígenas sobre alimentos y oro llevaron a Narváez y a sus hombres hacia el interior, hasta Apalache, donde se enfrentaron con sus habitantes. De allí marcharon hasta la aldea de Aute con la esperanza de encontrar más alimento y acercarse al mar. Hambrientos y enfermos, hostigados por los indios, fabricaron unas barcas con la pretensión de volver a México. Unos desaparecieron en el mar, otros naufragaron en las costas de Texas. De los cientos de hombres que formaban la expedición, sólo sobrevivieron para contarlo el propio Cabeza de Vaca, Alonso del Castillo Maldonado, 
Andrés Dorante y Esteban, el "negro árabe", según lo denomina Cabeza de Vaca. Por un tiempo conviven o se encuentran con otros compañeros náufragos. Después de varios años en la misma región, los cuatro echan a andar en busca de tierra donde hubiera españoles. El encuentro se produciría muy lejos de las costas de Florida, en tierras del actual estado mexicano de Sinaloa. Prácticamente habían caminado desde el Atlántico a las costas del Pacífico.

Alvar Núñez Cabeza de Vaca es el primer etnógrafo y el mayor andarín de América del Norte. Ambos oficios los ejerció a la fuerza, pero con buen talante y resignación. Es admirable, casi increíble, la abundancia y exactitud de detalles de su narración. Cabeza de Vaca y sus compañeros permanecieron casi siete años más o menos prisioneros de diversos grupos de indígenas que vivían en la costa e islas del sudoeste de Texas. Cuando se ponen en marcha, tomaron dirección sur en busca de la provincia de Pánuco, de la que estaban bastante más lejos de lo que suponían. Rectificaron y a partir de entonces caminaron hacia poniente. Cruzaron más de una vez el Río Grande y se introdujeron en la región de los indios pueblo. Por último, tomaron rumbo sur hasta llegar a la villa de Culiacán, en el extremo noroeste de México. Los cuatro náufragos habían caminado por los bordes de Texas, los límites de las Grandes Llanuras, la futura provincia de Nuevo México para penetrar por último en México por lo que hoy son los estados de Sonora y Sinaloa. Convivieron o conocieron, pues, tribus de indios karankawas y coahuiltecos, indios pueblo de Nuevo México, ópatas y pimas de Sonora.

Una vez en la ciudad de México, Cabeza de Vaca y sus compañeros de aventura presentaron ante la audiencia un escrito que se conoce como "Informe conjunto". Gonzalo Fernández de Oviedo conoció este texto y lo utilizó en su Historia general y natural de las Indias. De vuelta a España, Cabeza de Vaca escribió su Relación entre 1537 y 1540 . El texto se imprimió en Zamora en 1542. En 1555 hubo una segunda edición en Valladolid, acompañada de los Comentarios dedicados a su experiencia en el Río de la Plata. En esta segunda edición se le añadió título a cada capítulo, todos muy breves. Hubo nueva edición española en 1749 y otra en 1852. En el siglo $\mathrm{xx}$ han sido muchas las ediciones, algunas aparecidas en colecciones populares destinadas al lector de libros de aventura. Una reciente y asequible es la de Roberto Ferrando (1984), que incluye en un solo volumen la Narración y los Comentarios. En 1556 se publicó la Narración en italiano. En 1837 apareció la primera versión en francés. La primera traducción completa al inglés es de 1851. Cyclone Covey (1961) tradujo y publicó Adventures in the Unknown Interior of America teniendo a la vista las ediciones de 1542 y 1555 y el "Informe conjunto". Covey 
mantiene la división en capítulos, pero hace algunos arreglos que aumentan su número de treinta y ocho a cincuenta y siete. Más reciente es la edición de Martin A. Favata y José B. Fernández (1986) hecha a partir de la de Valladolid de 1555, aunque al final ofrecen "Notas de las variantes" halladas en la de Zamora. Rolena Adorno y Patrick Charles Pautz (1999) son autores de la más actual y completa edición de la Relación. Es una edición bilingüe a dos páginas que permite el contraste línea a línea. Adorno y Pautz han preferido la original de Zamora, más rara y desconocida, aunque analizan y reproducen en su estudio las variantes de la de Valladolid. Como ellos advierten, la primera versión obedecía a la urgencia de Cabeza de Vaca por informar al emperador de sus servicios y esperar otra oportunidad. La segunda está dirigida a un público cortesano amplio, por medio de una redacción más didáctica y literaria.

Todo lo que Cabeza de Vaca ve y cuenta era nuevo para el hombre europeo. Descubre tierras habitadas por indígenas que viven de la pesca, la caza, la recolección; cuando más, de una agricultura basada en el maíz. El hambre y el frío fueron las dos constantes de una aventura que en gran parte sucedió bajo fuertes lluvias, completamente desnudo o mal cubierto con pieles. Cuando todavía son unos cientos de hombres los que marchan por tierra, mientras los barcos costean, un español se ahoga con su caballo en la corriente de un río: "El caballo dio de cenar a muchos aquella noche ${ }^{1}$. La mayoría de los grupos indígenas que encontró Cabeza de Vaca vivían miserablemente. Sin embargo, compartieron en muchas ocasiones con los españoles sus pobres alimentos:

Otro día, saliendo el sol, que era la hora que los indios nos habían dicho, vinieron a nosotros, como lo habían prometido, y nos trajeron mucho pescado y de unas raíces que ellos comen, y son como nueces, algunas mayores o menores; la mayor parte de ellas se sacan de bajo del agua y con mucho trabajo. A la tarde volvieron y nos trajeron más pescado y de las misma raíces, y hicieron venir sus mujeres y hijos para que nos viesen, y así, se volvieron ricos de cascabeles y cuentas que les dimos.

El alimento más frecuente fueron las tunas, o higos chumbos, que los españoles habían conocido en las islas por el nombre que les daban los indios taínos de la Española:

Esta es una fruta que es del tamaño de huevos, y son bermejas y negras y de muy buen gusto. Cómenlas tres meses del año, en los cuales no comen otra cosa alguna...

${ }^{1}$ La selección de citas no sigue el orden de la narración, sino que se han agrupado con un cierto criterio temático. 
Cabeza de Vaca también describe la fauna que encuentra a su paso. Hablando del pueblo y tierra de Apalache enumera los siguientes animales:

... venados de tres maneras, conejos y liebres, osos y leones y otras salvajinas, entre las cuales vimos un animal que trae los hijos en una bolsa que en la barriga tiene. $\mathrm{Y}$ todo el tiempo que son pequeños los traen allí, hasta que saben buscar de comer. Y si acaso están fuera buscando de comer y acude gente, la madre no huye hasta que los ha recogido en su bolsa ${ }^{2}$.

De la fauna destaca el bisonte o búfalo, de momento nombrado con la palabra española más próxima:

\begin{abstract}
Alcanzan aquí vacas, y yo las he visto tres veces y comido de ellas, y paréceme que serán del tamaño de las de España; tienen los cuernos pequeños, y el pelo muy largo, merino; unas son pardillas, y otras negras, y a mi parecer tienen mejor y más gruesas carne que las de acá. De las que no son grandes hacen los indios mantas para cubrirse, y de las mayores hacen zapatos y rodelas; éstas vienen de hacia el Norte por la tierra adelante hasta la costa de la Florida, y tiéndense por toda la tierra más de cuatrocientas leguas; y en todo este camino, por los valles por donde ellas vienen, bajan las gentes que por allí habitan y se mantienen de ellas, y meten en la tierra grande cantidad de cueros.
\end{abstract}

Las circunstancias llevaron a Cabeza de Vaca y a sus compañeros a ejercer de médicos o curanderos. Las creencias mágicas de los indígenas los convirtieron en chamanes, término hoy universal para designar al especialista que en una tribu cura por medios naturales o por sugestión, hace llover, aleja o produce el mal. A la fuerza, fueron también por conveniencia los primeros chamanes no indígenas de las Américas. En alguna ocasión, Cabeza de Vaca actuó simplemente como cirujano y siempre, según su testimonio, confiando en la oración a falta de verdaderos conocimientos médicos. La fama de curanderos les precedió de tal modo que los indios les salían al encuentro:

En aquella isla que he contado nos quisieron hacer físicos sin examinarnos ni pedirnos títulos, porque ellos curan las enfermedades soplando al enfermo, y con aquel soplo y las manos echan de él la enfermedad. Y mandáronnos que hiciésemos lo mismo y sirviésemos en algo. Nosotros nos reíamos de ello diciendo que era burla y que no sabíamos curar, y por esto nos quitaban la comida hasta que hiciésemos lo que nos decían. [...] La manera con que nosotros curamos era santiguándolos y soplarlos, y rezarse un paternoster y un avemaría, y rogar lo mejor que podíamos a Dios nuestro Señor que les diese la salud y espirase [infundiese] en ellos que nos hiciesen algún buen tratamiento [...] Aquella misma noche que llegamos vinieron unos indios a Castillo, y dijéronle que estaban muy malos de la cabeza, rogándole que los curase; y después que los hubo santiguado y enco-

${ }^{2}$ La zarigüeya, mamífero americano de cola prensil. 
mendado a Dios, en aquel punto los indios dijeron que todo el mal se les había quitado. $\mathrm{Y}$ fueron a sus casas y trujeron muchas tunas y un pedazo de carne de venado, cosa que no sabíamos qué cosa era. Y como esto entre ellos se publicó, vinieron otros muchos enfermos en aquella noche a que los sanase, y cada uno traía un pedazo de venado; y tantos eran, que no sabíamos adonde poner la carne. Dimos muchas gracias a Dios porque cada día iba creciendo su misericordia y mercedes. Y después que se acabaron las curas comenzaron a bailar y hacer sus areitos y fiestas hasta otro día que el sol salió...

Las formas de vida de los indígenas aparecen con frecuencia en la narración con opiniones y juicios propios del autor:

Cuantos indios vimos desde la Florida aquí, todos son flecheros, y como son tan crecidos de cuerpo y andan desnudos, desde lejos parecen gigantes. Es gente a maravilla bien dispuesta, muy enjutos y de muy grandes fuerzas y ligereza. Los arcos que usan son gruesos como el brazo, de once o doce palmos de largo, que flechan a doscientos pasos con tan gran tiento, que ninguna cosa yerran. [...] A esta isla pusimos por nombre isla de Mal Hado. La gente que alli hallamos son grandes y bien dispuestos. No tienen otras armas sino flechas y arcos, en que son por extremo diestros. Tienen los hombres la una teta horadada por una parte a otra, y algunos hay que las tienen ambas, y por el agujero que hacen traen una caña atravesada, tan larga como dos palmos y medio, y tan gruesa como dos dedos. Traen también horadado el labio de abajo, y puesto en él un pedazo de caña delgada como medio dedo. Las mujeres son para mucho trabajo. La habitación que en esta isla hacen es desde octubre hasta en fin de febrero. El su mantenimiento es las raíces que he dicho, sacadas debajo el agua por noviembre y diciembre. Tienen cañales, y no tienen más peces de para este tiempo; de ahí adelante comen las raíces. En fin de febrero van a otras partes a buscar con qué mantenerse, porque entonces las raíces comienzan a nacer, y no son buenas. [...] Desde la isla de Mal Hado, todos los indios que hasta esta tierra vimos tienen por costumbre desde el día que sus mujeres se sienten preñadas no dormir juntos hasta que pasen dos años que han criado los hijos, los cuales maman hasta que son de edad de doce años, que ya entonces están en edad que por sí saben buscar de comer. Preguntámosles que por qué los criaban así, y decían por la mucha hambre que en la tierra había, que acontecía muchas veces, como nosotros veíamos, estar dos o tres días sin comer, y a las veces cuatro; y por esta causa los dejaban mamar, porque en los tiempos de hambre no muriesen. [...] Todos estos [indios] acostumbran dejar sus mujeres cuando entre ellos no hay conformidad, y se tornan a casar con quien quieren. Esto es entre los mancebos, mas los que tienen hijos permanecen con sus mujeres y no las dejan.

La vocación etnográfica de Cabeza de Vaca se observa en su interés por describir las gentes y sus costumbres y su propósito por dejar constancia:

Esto he querido contar, porque allende que todos los hombres desean saber las costumbres y ejercicios de los otros, los que algunas veces se vinieren a ver con ellos estén avisados de sus costumbres y ardides, que suelen no poco aprovechar 
en semejantes casos. [...] También quiero contar sus naciones y lenguas, que desde la isla de Mal Hado hasta los últimos hay. En la isla de Mal Hado hay dos lenguas, a los que unos llaman de Caoques y a los otros llaman de Han. En la tierra firme, enfrente de la isla, hay otros que se llaman de Chorruco, y toman el nombre de los montes donde vine. Adelante, en la costa del mar, habitan otros que se llaman Doguenes, y enfrente de ellos otros que tienen por nombre los de Mendica. Más adelante, en la costa, están los Quevenes, y enfrente de ellos, dentro en la tierra firme, los Mariames; y yendo por la costa adelante, están otros que se llaman Guaycones, y enfrente de éstos, dentro en la tierra firme, los Iguaces. Cabo de éstos están otros que se llaman Atayos, y detrás de éstos, otros, Acubadaos, y de éstos hay muchos por esta vereda adelante. En la costa viven otros llamados Quitoles, y enfrente de esto, dentro en la tierra firme, los Avavares. Con éstos se juntan los Maliacones, y otros Cutalchiches, y otros que se llaman Susolas, y otros que se llaman Comos, y adelante en la costa están los Camoles, y en la misma costa adelante, otros a quienes nosotros llamamos los Higos. Todas estas gentes tienen habitaciones y pueblos y lenguas diversas. Entre éstos hay una lengua en que llaman a los hombres por mira acá, arre acá; a los perros, xo. En toda la tierra se emborrachan con humo [del peyote], y dan cuanto tienen por él. Beben también otra cosa que sacan de las hojas de los árboles, como de encina, y tuéstanla en unos botes al fuego, y después que la tienen tostada hinchan el bote de agua, y así lo tienen sobre el fuego, y cuando ha hervido dos veces, échanlo en una vasija y están enfriándola con media calabaza, y cuando está con mucha espuma bébenla tan caliente cuanto pueden sufrir. [...] En el tiempo que así estaba, entre éstos vi una diablura, y es que vi un hombre casado con otro, y éstos son unos hombres amarionados, impotentes, y andan tapados como mujeres y hacen oficio de mujeres, y tiran arco y llevan muy gran carga, entre éstos vimos muchos de ellos así amarionados como digo, y son más membrudos que los otros hombres y más altos; sufren muy grandes cargas.

El valor etnográfico del relato de Cabeza de Vaca se apreciará mejor teniendo en cuenta varias consideraciones. La tierra que cruzan les es absolutamente desconocida. A esta ignorancia de la geografía se suma la barrera de la incomunicación con los indios que encuentran en su camino. Muy al principio, mientras unos marchan a pie y otros costean, dice Cabeza de Vaca:

... sobre todo, íbamos mudos y sin lengua, por donde mal nos podíamos entender con los indios, ni saber lo que de la tierra queríamos, y que entrábamos por tierra de que ninguna relación teníamos, ni sabíamos de qué suerte era, ni lo que en ella había, ni de qué gente estaba poblada, ni a qué parte de ella estábamos.

Casi al final de la gran caminata, Cabeza de Vaca se refiere de nuevo al problema de la comunicación, aunque ahora cuenta con la experiencia de varios años entre indígenas:

Pasamos por gran número y diversidades de lenguas; con todas ellas Dios nuestro Señor nos favoreció, porque siempre nos entendieron y les entendimos. Y así, 
preguntábamos y respondían por señas, como si ellos hablaran nuestra lengua y nosotros la suya, porque aunque sabíamos seis lenguas, no nos podíamos en todas partes aprovechar de ellas, porque hallamos más de mil diferencias.

Si el espacio recorrido fue casi infinito, el tiempo no lo sería menos tanto en su percepción como en duración absoluta. Todavía en la isla de Mal Hado, Cabeza de Vaca escribe:

Mi enfermedad estorbó que no les pude seguir [a otros españoles que habían aparecido] ni los vi. Yo hube de quedar con estos mismos indios de la isla más de un año, y por el mucho trabajo que me daban y mal tratamiento que me hacían, determiné de huir de ellos y irme a los que moran en los montes y tierra firme...

No mejoró mucho la situación con el cambio, debido a la pobreza y rigor de la naturaleza, aunque los indígenas que mantenían hostilidades entre sí encontraron en el español un útil mercader. A unos les llevaba caracolas y conchas del mar, a otros productos de la tierra como cueros, almagra para sus pinturas, pedernales y cañas duras para sus flechas:

Los trabajos que en esto pasé sería largo contarlos, así de peligros y hambres como de tempestades y fríos. [...] Fueron casi seis años el tiempo que yo estuve en esta tierra solo entre ellos y desnudo, como todos andaban. La razón por que tanto me detuve fue por llevar conmigo un cristiano que estaba en la isla, llamado Lope de Oviedo [...] y por sacarlo de allí yo pasaba a la isla cada año y le rogaba que nos fuésemos a la mejor maña que pudiésemos en busca de cristianos. Y cada año me detenía diciendo que el otro siguiente nos iríamos.

Tiempo después, en plena dedicación a la medicina, dice Cabeza de Vaca:

Nosotros estuvimos con aquellos indios avavares ocho meses, y esta cuenta hacíamos por las lunas.

Espacio y tiempo quedan resumidos en las últimas páginas del relato, cuando el autor invoca a Dios y al rey:

Dios nuestro Señor, por su infinita misericordia, quiera que en los días de Vuestra Majestad y debajo de vuestro poder y señorío, estas gentes vengan a ser verdaderamente y con entera voluntad sujetas al verdadero Señor que las crió y redimió, lo cual tenemos por cierto que así será, y que Vuestra Majestad ha de ser el que lo ha de poner en efecto, que no será tan difícil de hacer, porque dos mil leguas que anduvimos por tierra y por la mar en las barcas, y otros diez meses que después de salidos de cautivos, sin parar, anduvimos por la tierra, no hallamos sacrificios ni idolatrías. En este tiempo atravesamos de una mar a otra, y por la noticia que con mucha diligencia alcanzamos a entender, de una costa a la otra, por lo más ancho, puede haber doscientas leguas. $\mathrm{Y}$ alcanzamos a entender que en la costa del sur hay perlas y mucha riqueza, y que todo lo mejor y más rico está cerca de ella. 
El relato de Cabeza de Vaca es de principio a fin una declaración de fe y confianza en Dios. Ruega y da gracias al Señor continuamente, y se consuela con el recuerdo de la Pasión de Cristo. Es un hombre de su tiempo y de su cultura, que encuentra en la fe la fuerza para sobrevivir. Durante ocho años, su objetivo es llegar a tierra conocida y encontrarse con españoles, pero en ningún caso los nombra con este término, sino el de "cristianos", que en aquellas circunstancias eran sinónimos. Junto a su fe religiosa, muestra su fidelidad al emperador y su identificación con la política oficial. Otro rasgo de la personalidad de Cabeza de Vaca, compartido con otros muchos españoles de la época, es su pasión por la aventura y la gloria. Vuelto a España, fue nombrado gobernador del Río de la Plata. El hombre que había caminado casi de mar a mar por América del Norte, marchó a pie desde las costas de América del Sur hasta Asunción del Paraguay, y en el camino, descubrió las cataratas de Iguazú. Las intrigas y hostilidades entre españoles, tan abundantes en la empresa hispana, le llevaron al encarcelamiento. Su segundo regreso a España fue triste y humillante, pues volvió encadenado. Fue juzgado y condenado a ocho años de destierro en África. Pleiteó ante la Corona y, finalmente, fue absuelto y nombrado juez en Sevilla. Un final mediocre para un hombre que había vivido una aventura increíble.

Es tiempo de resumir y terminar. En el caso que he utilizado hay dos grandes realidades, ambas increibles o muy difícil de creer si no estuvieran documentadas. Una es la propia biografía del autor, quien voluntariamente participó en una desgraciada expedición, pero ni escarmentado ni satisfecho con su experiencia por América del Norte, cruza de nuevo el océano y realiza otra tremenda caminata desde las costas de Brasil hasta el corazón del Paraguay. ¿Por qué insistió Cabeza de Vaca en su experiencia americana? ¿Qué le movió a una nueva aventura en las Indias? ¿Por qué tantos otros españoles persistieron en sus ambiciones y corrieron la misma o peor suerte? La otra realidad increíble es la aventura vivida por Cabeza de Vaca en lo que fue el primer trabajo de campo realizado por nadie en América del Norte.

Cabeza de Vaca ha sido calificado con todo merecimiento como el primer etnógrafo de lo que hoy son los Estados Unidos de América. Ciertamente, su experiencia de campo fue involuntaria, forzada, como también lo fue en el siglo xx la de Bronislaw Malinowski cuando las circunstancias de la primera guerra mundial le confinaron en las Islas Trobriand debido a su origen polaco, que le hacía sospechoso ante los británicos. Esta especie de encierro le dio ocasión y tiempo para escribir después su famosa obra Argonautas del Pacífico Occidental (1979), modelo de etnografía para muchas generaciones de estudiantes de antropo- 
logía. Pero hay también grandes diferencias entre Cabeza de Vaca y Malinowski: una distancia de cuatro siglos; una experiencia de campo de ocho años frente a un total de dieciocho meses en dos períodos; una ignorancia absoluta del terreno por parte del español, que se movió siempre por regiones no pisadas por ningún europeo. Pero lo que llama más la atención es la enorme resistencia física y psíquica de Cabeza de Vaca, su voluntad de sobrevivir y encontrarse con los suyos. Y en el aspecto científico o académico, lo más significativo es su obra escrita, su descripción de la naturaleza y de sus habitantes, su economía, costumbres y creencias. Es decir, la cultura del "otro".

El escritor inglés Daniel Defoe (1660-1731) nos dejó una novela tan popular que el nombre del protagonista se ha convertido en término universal para expresar una forma de ser y de vivir. La permanencia de un marinero escocés durante cinco años en una isla absolutamente desierta (Islas de Juan Fernández, en el Pacífico Sur), parece que inspiró a Defoe su Robinson Crusoe. Cabeza de Vaca hizo también literatura al plasmar su aventura en un libro, pero su texto transcribe una realidad más larga y no menos dura que él vivió en su carne y sintió en su mente. El libro de Cabeza de Vaca nos habla de unas gentes que existieron y perduraron, como han podido conocer y estudiar siglos después los antropólogos a través de sus descendientes. La Relación, o Naufragios, de Cabeza de Vaca es una combinación de literatura y etnografía. Es decir, etnoliteratura. El texto es una obra literaria donde no hay ficción, no hay invención, aunque lo que se cuenta resulte increíble. Es una obra híbrida, como un puente entre etnografía y literatura, entre experiencia vivida y realidad imaginada. Ante obras como ésta surgen interrogantes que hacen pensar y, sobre todo, invitan a intentar nuevos caminos metodológicos. ¿Por qué no acudir de manera sistemática, selectiva y rigurosa a la literatura como fuente etnográfica si hay textos que son las dos cosas al mismo tiempo, realidad y ficción, experiencia propia y experiencia ajena? En el caso de Cabeza de Vaca, el contenido de su obra es tan extraordinario que parece fantástico. ¿Dónde están los límites, o la diferencia, entre la realidad y la ficción, entre la verdad y la mentira, entre lo posible y lo imposible, entre lo sucedido y lo imaginado, entre la realidad observada y la interpretación del científico social? ¿Qué disciplina o ciencia, qué individuo o escuela puede establecer tales límites o fronteras cuando, a veces, la realidad supera a la ficción, la intuición a la comprobación empírica, la imaginación al trabajo de laboratorio, la filosofía y la poesía a la ciencia? 


\section{BIBLIOGRAFÍA CITADA}

Adorno, Rolena y Patrick Charles Pautz. 1999. Alvar Núñez Cabeza de Vaca. His account, his life, and the expedition of Pánfilo de Narváez. Lincoln: University of Nebraska Press.

Cover, CyClone (trad. y ed.). 1961. Cabeza de Vaca's adventures in the unknown interior of America. Alburquerque: University of New Mexico Press.

FAvATA, MARTIN y José B. Fernández (eds.). 1986. La Relación o Naufragios de Alvar Núñez Cabeza de Vaca. Potomac, Maryland: Scripta Humanistica.

FERRANDO, ROBERTO (ed.). 1984. Alvar Núñez Cabeza de Vaca. Naufragios y Comentarios. Madrid: Historia 16.

GEERTZ, CLIFFORD. 1983. Local knowledge. Further essays in interpretive antbropology. Nueva York: Basic Books, Inc.

- 1989. El antropólogo como autor. Barcelona: Paidós.

JiMÉNEZ NÚÑEZ, ALFREDO. 1994. "Fuentes y métodos de la antropología: consideraciones un tanto críticas", en Manuel de la Fuente Lombo (ed.), Etnoliteratura. Un nuevo método de análisis en antropología: 9-49. Córdoba: Servicio de Publicaciones de la Universidad de Córdoba.

- 1997. "La novela como etnografía: del comisario Maigret a Los santos inocentes", en Manuel de la Fuente y M. a Ángeles Hermosilla (eds.), Etnoliteratura: ¿Una antropología de lo imaginario?: 105-121. Córdoba: Servicio de Publicaciones de la Universidad de Córdoba.

MALINOWSKI, BRONISLAW. 1979 [1922]. Argonautas del Pacífico Occidental. Barcelona: Península. 